\title{
Duodenum/Ampulla/Jejunum/Ileum Neuroendocrine Tumor pNO TNM Finding v7
}

National Cancer Institute

\section{Source}

National Cancer Institute. Duodenum/Ampulla/Jejunum/lleum Neuroendocrine Tumor

pNO TNM Finding v7. NCI Thesaurus. Code C90093.

Duodenum/ampulla/jejunum/ileum neuroendocrine tumor with no regional lymph node metastasis. (from AJCC 7th Ed.) 